\title{
Schadstoffe im Grundwasser: aktuelle Herausforderungen
}

\author{
Andre Banning $^{1}$ (i) $\cdot$ Helena Banning ${ }^{2}$ \\ Eingegangen: 19. November 2018 / Online publiziert: 16. Januar 2019 \\ (c) Springer-Verlag GmbH Deutschland, ein Teil von Springer Nature 2019
}

Die Ressource Grundwasser ist einer Vielzahl von geogenen und anthropogenen Stoffeinträgen ausgesetzt. Inzwischen sind zahlreiche potenziell öko- und/oder humantoxikologisch relevante organische und anorganische Stoffe bekannt, die die Qualität des Grundwassers mindern und die Erfüllung wichtiger ökologischer und wasserwirtschaftlicher Funktionen beeinträchtigen. Beispielhaft sind aus dem anthropogenen Bereich die vieldiskutierte Nitratproblematik, sowie Einträge von Pflanzenschutzmitteln, Bioziden und Industriechemikalien bzw. deren Abbauprodukte zu nennen. Hierbei sind sowohl technische als auch regulatorische Herausforderungen zu verzeichnen, die sich etwa aus disziplinär unterschiedlichen Ansätzen und teils inkonsistenten Regelungen in Umweltschutz-, Trinkwasser- und Chemikalienrecht ergeben. Aus verschiedensten geogenen Quellen leiten sich großflächige Belastungen mit beispielsweise Fluorid, Arsen oder Uran ab. Die rasche Entwicklung der jungen, stark interdisziplinären „Medical Geology“ (Erforschung der Beziehungen zwischen geologischen Faktoren und menschlicher Gesundheit) dokumentiert die steigende internationale Aufmerksamkeit für derartige Phänomene.

Das Thema Grundwasserschutz ist mit der Verabschiedung der Sustainable Development Goals (SDG) im Jahr 2015 auf höchster politischer Ebene angekommen, wobei mit dem SDG Nr. 6 nicht nur die Bereitstellung von sauberem Trinkwasser, sondern auch der Schutz aller Grundwasserkörper selbst festgeschrieben ist. Daraus ergeben sich mannigfaltige Herausforderungen sowie ein bedeutender

Andre Banning

andre.banning@rub.de

Helena Banning

helena.banning@uba.de

1 Lehrstuhl Angewandte Geologie, Ruhr-Universität Bochum, Universitätsstraße 150, 44801 Bochum, Deutschland

2 Fachgebiet Pflanzenschutzmittel, Umweltbundesamt, Wörlitzer Platz 1, 06844 Dessau-Roßlau, Deutschland
Forschungs- und Handlungsbedarf. Dies umfasst u.a. die Identifikation von Quellen und Mobilisierungsmechanismen, das Verständnis von Transport-, Abbau- und Akkumulationsprozessen, die Verbesserung von Laboranalytik und numerischer Modellierung, Strategien zu Risikomanagement, Vermeidung und Substitution problematischer Stoffe, Sanierungsansätze, Ableitung von Grenzwerten sowie Empfehlungen für den Gewässerschutz.

Deshalb freuen wir uns über das große Interesse am Aufruf zur Manuskripteinreichung und über die Vielfalt und Interdisziplinarität der publizierten Artikel. Das vorliegende Themenheft zeigt einen Querschnitt durch die aktuellen Fragestellungen und Herausforderungen im Bereich Grundwasserkontamination, sowohl aus Sicht der Grundlagenund Anwendungsforschung als auch der hydrogeochemischen und regulatorischen Praxis und der Grundwassersanierung. Es wird ein breites Spektrum an Schadstoffen geogen, anthropogen, organisch, anorganisch - und methodischen Ansätzen wie Labor-, Gelände-, Monitoring- und Modellierungsstudien abgedeckt und miteinander kombiniert. Die Skala reicht dabei vom Einzelmolekül bis zum kompletten Bundesland. Neben den Beiträgen im aktuellen Heft werden zwei weitere Artikel aus dem Themenkreis „Schadstoffe im Grundwasser“ in Heft 2/2019 erscheinen.

In Zukunft wird das Thema „Schadstoffe im Grundwasser" für weitere Herausforderungen sorgen, die inter- und transdisziplinäre sowie internationale Kooperation von Wissenschaftlern und Praktikern aus den Bereichen Geowissenschaften, Ökologie, Ingenieurwesen, Medizin, Gesellschaftswissenschaften, Politik und vielen anderen erfordert.

Wir wünschen Ihnen eine aufschlussreiche und spannende Lektüre. 